\title{
Above band gap absorption spectra of the arsenic antisite defect in low temperature grown GaAs and AIGaAs
}

Dankowski, S. U.; Streb, D.; Ruff, M.; Kiesel, P.; KneissI, M.; Knüpfer, B.; Döhler, G. H.; Keil, Ulrich Dieter Felix; Sørensen, Claus Birger; Verma, A. K.

Published in:

Applied Physics Letters

Link to article, DOI:

10.1063/1.116748

Publication date:

1996

Document Version

Publisher's PDF, also known as Version of record

Link back to DTU Orbit

Citation (APA):

Dankowski, S. U., Streb, D., Ruff, M., Kiesel, P., Kneissl, M., Knüpfer, B., Döhler, G. H., Keil, U. D. F., Sørensen, C. B., \& Verma, A. K. (1996). Above band gap absorption spectra of the arsenic antisite defect in low temperature grown GaAs and AlGaAs. Applied Physics Letters, 68(1), 37-39. https://doi.org/10.1063/1.116748

\section{General rights}

Copyright and moral rights for the publications made accessible in the public portal are retained by the authors and/or other copyright owners and it is a condition of accessing publications that users recognise and abide by the legal requirements associated with these rights.

- Users may download and print one copy of any publication from the public portal for the purpose of private study or research.

- You may not further distribute the material or use it for any profit-making activity or commercial gain

- You may freely distribute the URL identifying the publication in the public portal 


\title{
Above band gap absorption spectra of the arsenic antisite defect in low temperature grown GaAs and AIGaAs
}

\author{
S. U. Dankowski, ${ }^{\text {a) }}$ D. Streb, M. Ruff, P. Kiesel, M. Kneissl, B. Knüpfer, and G. H. Döhler \\ Institut für Technische Physik I, Friedrich-Alexander-Universität Erlangen-Nürnberg, \\ Erwin-Rommel-Strasse 1, 91058 Erlangen, Germany \\ U. D. Keil and C. B. Sorenson \\ Mikroelecktonik Centret, DTH building 345 east, 2800 Lyngby, Denmark
}

\begin{abstract}
A. K. Verma
Department of Electrical Engineering \& Computer Science, University of California, Berkeley, California 94720
\end{abstract}

(Received 21 September 1995; accepted for publication 21 October 1995)

\begin{abstract}
Room temperature absorption spectra of low temperature molecular beam epitaxy grown GaAs (LT-GaAs) and AlGaAs (LT-AlGaAs) are reported. We performed measurements in an extended spectral range from $0.8 \mathrm{eV}$ to photon energies of $2.8 \mathrm{eV}$ far above the band gap. For as-grown LT-materials, the absorption coefficients at the band gap are twice as high as for high temperature grown materials. By annealing the samples, we obtained a drastic reduced absorption coefficient below as well as above the band gap. We observed absorption changes up to $17000 \mathrm{~cm}^{-1}$ for LT-GaAs and $9000 \mathrm{~cm}^{-1}$ for LT-AlGaAs taking place in a two phase process. (C) 1996 American Institute of Physics. [S0003-6951(96)00201-9]
\end{abstract}

Growth of GaAs at low substrate temperatures ${ }^{1}$ was first reported by Smith et al. in 1988. Since then many articles have been published demonstrating the unusual and very appealing properties of this material, such as femtosecond recombination lifetimes for photoinduced carriers in the asgrown state $^{2}$ and semi-insulating behavior with a sheet resistance up to $10^{7} \Omega \mathrm{cm}$ after annealing ${ }^{3}$ (typically around $600{ }^{\circ} \mathrm{C}$ for several minutes), respectively. These remarkable features make low temperature (LT)-GaAs and related materials like LT-AlGaAs very attractive for ultrafast optoelectronic applications.

Molecular beam epitaxy (MBE) growth at low substrate temperatures $\left(180-250{ }^{\circ} \mathrm{C}\right)$ and large As/Ga flux ratios results in up to $1 \%$ excess As in LT materials. ${ }^{4}$ Due to the reduced surface mobility and the excess of As, arsenic antisite $\left(\mathrm{As}_{\mathrm{Ga}}\right)$ and arsenic interstitial $\left(\mathrm{As}_{i}\right)$ defects $\left(\approx 10^{20}\right.$ $\mathrm{cm}^{-3}$ ) are created.

The $\mathrm{As}_{\mathrm{Ga}}$ defect shows similarities to the well-known EL2 defect and has been investigated in detail by a large number of scientists (see, e.g., Ref. 5), but a lack of clarity still remains about the atomic structure of this defect and the mechanism of occupying a metastable state at temperatures below $130 \mathrm{~K}$. Nevertheless, a promising theoretical model has been presented. ${ }^{6,7}$

One common technique for characterizing the EL2 defect is to perform subband gap infrared absorption (IRA) experiments. Comparing the results of experiments on semiinsulating substrates grown by the liquid encapsulated Czochralski (LEC) method (containing about $10^{16} \mathrm{~cm}^{-3}$ EL2 defects) and those obtained on LT-GaAs layers, similarities in absorption spectra ${ }^{4}$ and the metastable behavior ${ }^{8}$ were observed. These similarities were confirmed by comparing various other properties of the EL2 defect and LT-GaAs defects

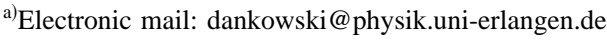

using a variety of experimental techniques. ${ }^{9,10}$ Certain deviations of conformity in those spectra have been explained by local strain in LT-GaAs. ${ }^{4}$ Taking these strain effects into account, it seems reasonable to call at least a certain part of the defects in LT-GaAs. "EL2-like defects."

In IRA experiments on semi-insulating LEC-grown GaAs substrates, the EL2-like $\mathrm{As}_{\mathrm{Ga}}$ defect spectra can be observed only at photon energies less than the band gap of the host material ( $1.42 \mathrm{eV}$ at room temperature). Above the band gap, their contribution to the absorption coefficient $\left(<10 \mathrm{~cm}^{-1}\right)^{11}$ cannot be discriminated from the strong bandto-band absorption. However, this is not the case in IRA experiments on LT-GaAs. In this material, the defect-induced absorption is almost four orders of magnitude higher than in semi-insulating material due to the extremely high defect density. Therefore, the observable spectral region for EL2defect absorption can be extended to photon energies above band gap. This is possible for these energies because the above band gap absorption caused by deep defects in LTGaAs is of the same order of magnitude as the absorption caused by the host material. Hence, it enables us to observe above band gap absorption spectra of the EL2-like defects in thin layers of LT-GaAs and LT-AlGaAs.

In this letter we present absorption spectra for photon energies from 0.8 to $2.8 \mathrm{eV}$ far above the band gap using the following three samples:

(i) Sample A consists of a GaAs buffer layer and a $50 \mathrm{~nm}$ thick AlAs sacrificial layer grown on a semi-insulating GaAs substrate followed by a $1.3 \mu \mathrm{m}$ thick undoped LT-GaAs layer grown at $250{ }^{\circ} \mathrm{C}$.

(ii) Sample B is the reference sample for A and possesses a similar structure with $1.5 \mu \mathrm{m}$ standard GaAs grown at $580^{\circ} \mathrm{C}$ instead of LT-GaAs.

(iii) Sample $\mathrm{C}$ is comprised of a $1 \mu \mathrm{m}$ thick undoped LT$\mathrm{Al}_{0.3} \mathrm{Ga}_{0.7}$ As layer $\left(T_{\text {growth }}=250{ }^{\circ} \mathrm{C}\right)$ and a GaAs buffer layer grown on a semi-insulating GaAs substrate. 


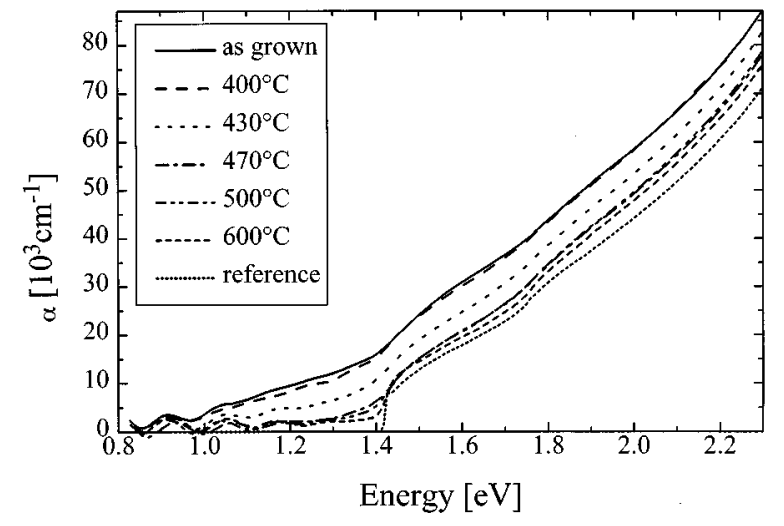

FIG. 1. Absorption spectra of LT-GaAs grown at $250{ }^{\circ} \mathrm{C}$ and annealed at different temperatures. In addition, the spectrum of the GaAs reference sample is shown.

In order to observe the influence of annealing on the absorption, we tempered pieces of sample A at 400, 430, 470,500 , and $600{ }^{\circ} \mathrm{C}$ in a rapid thermal annealer under $\mathrm{N}_{2}$ atmosphere for $1 \mathrm{~min}$. By annealing the LT-GaAs samples, the EL2-like $\mathrm{As}_{\mathrm{Ga}}$ antisite defects precipitate into As clusters. $^{12}$ To avoid As evaporation during annealing, the samples were placed face down on a GaAs substrate. One piece of sample $\mathrm{C}$ was annealed at $600{ }^{\circ} \mathrm{C}$ using the same procedure.

After annealing the samples, we separated the thin epitaxial LT-GaAs and standard GaAs layers of samples A and $\mathrm{B}$ from the substrate by epitaxial liftoff (ELO). ${ }^{13}$ The ELO was done by using $10 \% \mathrm{HF}$, which etches the AlAs sacrificial layer highly selective against GaAs. To obtain a thin LTAlGaAs layer, we polished the substrate of the pieces of sample C down to roughly $100 \mu \mathrm{m}$ and used a selective etchant based on citric acid and hydrogen peroxide ${ }^{14}$ to remove the remaining substrate.

First transmission measurements on these samples have shown that the absorption spectra are strongly distorted by oscillations for energies below the band gap that are attributed to the air-LT-GaAs (LT-AlGaAs)-glass Fabry-Pérot cavity. With increasing annealing temperature, the extrema of the Fabry-Pérot oscillations (FPO) shift towards higher energies. This result confirms our previously reported annealing induced decrease of the refractive index $\Delta n \approx 0.2$ in LT-GaAs. ${ }^{15}$ For the following measurements, we suppressed the FPO for photon energies near the band gap by evaporating an antireflection coating onto the LT-(Al)GaAs surfaces.

After preparing the samples, room temperature transmission measurements were performed from $0.8 \mathrm{eV}$ to photon energies far above the band gap. To obtain the absorption coefficient, we compared the optical power transmitted through the setup with and without sample after correcting the data for the reflection losses.

Figure 1 shows the absorption spectra of the various annealed pieces of sample A and the spectra of the reference sample. The observable negative absorption coefficients are artificially created by FPO at photon energies where the antireflection coating is not effective anymore.

We notice the well-known ${ }^{8}$ subband gap absorption of

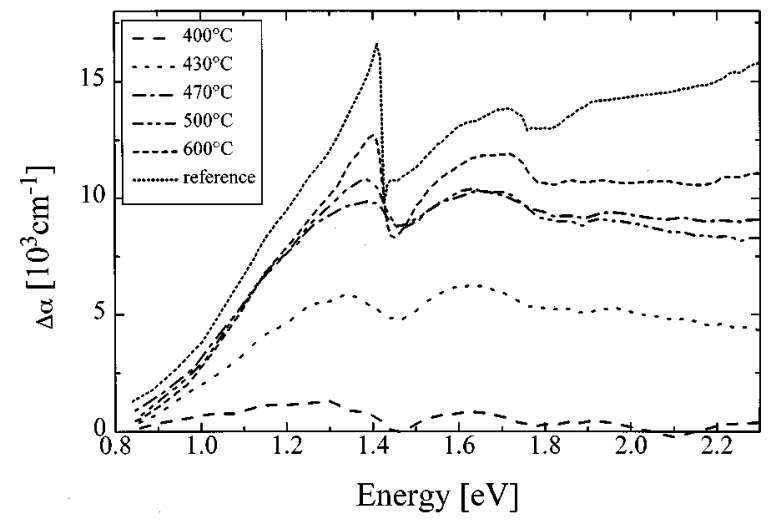

FIG. 2. Absorption change $\Delta \alpha$ of various annealed LT-GaAs samples relative to the as-grown sample. The effects due to the FPO below the band gap were eliminated in the curves.

LT-GaAs for photon energies below the band gap. These photons are absorbed solely by the immense amount of deep defects formed by excess As. This absorption can be decreased by more than one order of magnitude due to the annealing induced agglomeration of the $\mathrm{As}_{\mathrm{Ga}}$ antisite defects into As precipitates. However, for higher photon energies, both interband as well as defect absorption contribute to the absorption of LT-GaAs. The latter contribution is the difference between the absorption coefficients of the as-grown piece of sample A and the reference sample. Also, for photon energies above the band gap, the defect absorption is strongly reduced by annealing due to the reduction of $\mathrm{As}_{\mathrm{Ga}}$ antisite defects. To our knowledge these are the first above band gap absorption data of LT-GaAs.

Figure 2 shows the annealing induced absorption changes $\Delta \alpha$ of LT-GaAs for various annealing temperatures determined by subtraction of the as-grown spectra from the other curves of Fig. 1. The change of the absorption coefficient follows a two-phase process: (1) For annealing temperatures between 400 and $470{ }^{\circ} \mathrm{C}$, we observe a significant absorption change $\Delta \alpha$ of about $10000 \mathrm{~cm}^{-1}$ for photon energies around the band gap. This is due to the annealing induced reduction of the defect concentration. For these annealing temperatures, the absorption changes show only weak structures. Only for photon energies around the band gap there is a slight dip in the spectra. (2) For higher annealing temperatures, there is almost no further increase of $\Delta \alpha$, but the structure around the band gap becomes more pronounced. In particular, the difference spectrum between the as-grown sample and the reference sample exhibits a sharp peak of almost $7000 \mathrm{~cm}^{-1}$. The comparison of the absorption spectra of Fig. 1 shows that this structure results from the flat slope of the absorption coefficient of as-grown LT-GaAs near the band edge, which is getting sharper by annealing.

The $\Delta \alpha$ spectra of the $600{ }^{\circ} \mathrm{C}$ annealed sample $\mathrm{A}$ and the reference sample exhibit another, much smaller, dip of $\Delta \alpha$ at photon energies around $1.75 \mathrm{eV}$. At this energy the splitoff band to conduction band transition sets in. Therefore, this structure is also caused by the flat slope of the absorption coefficient of as-grown LT-AlGaAs near the splitoff transition, which is getting sharper by annealing. Figure 3 shows 


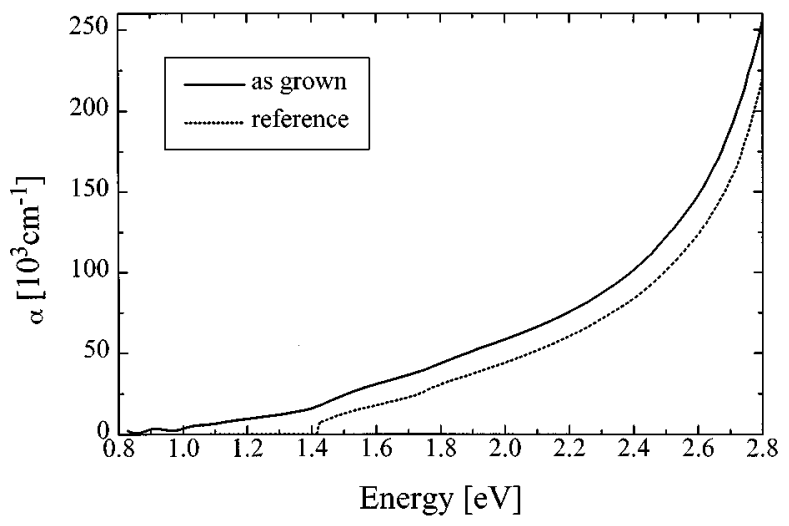

FIG. 3. Extended absorption spectra of the as-grown LT-GaAs sample and the GaAs reference sample. For energies above $1.8 \mathrm{eV}$, no obvious structure is visible.

an extended plot of the absorption spectra for as-grown LTGaAs and standard GaAs. The spectra exhibit a structureless shape for photon energies above $1.8 \mathrm{eV}$, while the difference in absorption between as-grown LT-GaAs and standard GaAs is nearly constant. For energies above $2.3 \mathrm{eV}$, the experimental error is getting too large for calculating the absorption change $\Delta \alpha$, while the tendency is described correctly.

A slightly different behavior is observed for the LTAlGaAs samples shown in Fig. 4. Sample $\mathrm{C}$ exhibits a similar tendency in the absorption spectra as LT-GaAs. For this sample, the additional defect absorption is considerably smaller $\left(10000 \mathrm{~cm}^{-1}\right.$ near the band gap), and its set in is shifted to higher energies due to the different band gap.

The absorption change between the as-grown sample and the sample annealed at $600{ }^{\circ} \mathrm{C}$ shown in Fig. 5 reaches its maximum of $9000 \mathrm{~cm}^{-1}$ around the band gap energy followed by a sharp dip down to $2000 \mathrm{~cm}^{-1}$ at $1.82 \mathrm{eV}$. The subsequent decrease of the absorption change results in a $\Delta \alpha$ of about $4000 \mathrm{~cm}^{-1}$ at $2.3 \mathrm{eV}$. Also for this material, an absorption peak is observed around the band gap. Again, this peak and the following dip is caused by the flat slope of the absorption coefficient of as-grown LT-AlGaAs near the band gap energy which is getting sharper by annealing.

In conclusion, we investigated to our knowledge for the first time the above band gap absorption of LT-GaAs and

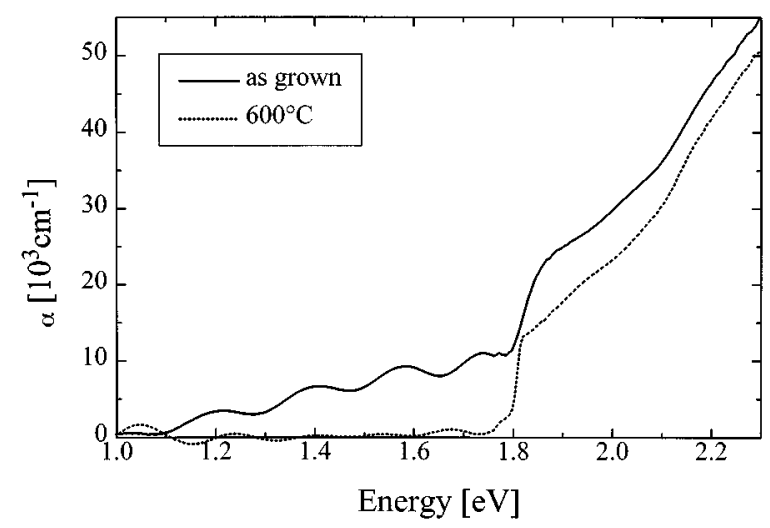

FIG. 4. Absorption of as-grown and $600{ }^{\circ} \mathrm{C}$ annealed LT-AlGaAs samples.

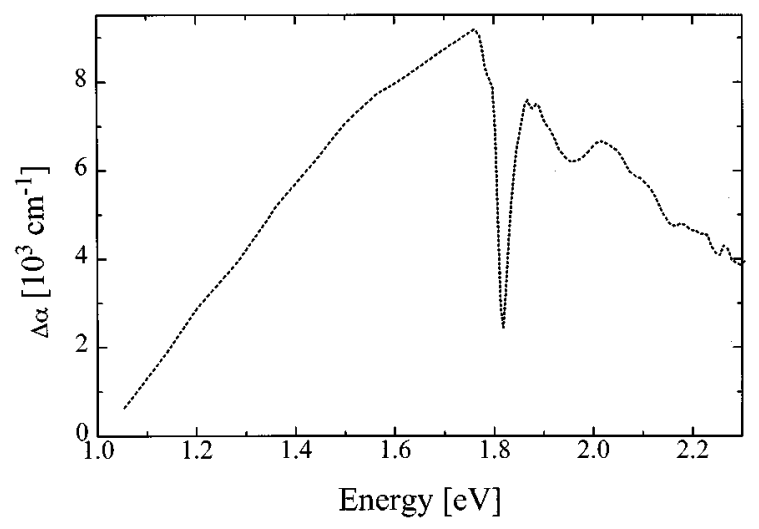

FIG. 5. Absorption change of the $600{ }^{\circ} \mathrm{C}$ annealed LT-AlGaAs samples relative to the as-grown sample. The effects due to the FPO below the band gap were eliminated in the curves.

LT-AlGaAs at room temperature. Compared to the reference sample, we observed an increased absorption coefficient below as well as above band gap energies. The annealing induced absorption change amounts to up to $17000 \mathrm{~cm}^{-1}$ for LT-GaAs and $9000 \mathrm{~cm}^{-1}$ for LT-AlGaAs, respectively. The change of the absorption coefficients of LT-GaAs is a twophase process. Up to $470{ }^{\circ} \mathrm{C}$ there is a strong decrease of $\alpha$ with only weak structures. For higher annealing temperatures, a sharp structure appears at band gap energy as well as a much smaller one at the energy of the splitoff band to conduction band transition. These structures are even more strongly developed in the difference spectrum of the asgrown LT-GaAs sample and reference sample. A similar behavior was also observed for LT-AlGaAs but with smaller $\Delta \alpha$ and shifted to the corresponding band gap.

The project is partly founded by the Deutsche Forschungsgemeinschaft.

${ }^{1}$ F. W. Smith, A. R. Calawa, C.-L. Chen, M. J. Manfra, and L. J. Mahoney, IEEE Electron Device Lett. 9, 2 (1988).

${ }^{2}$ F. W. Smith, H. Q. Lee, V. Diadiuk, M. A. Hollis, A. R. Calawa, S. Gupta, M. Frankel, D. R. Dykaar, G. A. Mourou, and T. Y. Hsiang, Appl. Phys. Lett. 54, 890 (1989).

${ }^{3}$ A. K. Verma, J. Tu, J. S. Smith, H. Fujioka, and E. R. Weber, J. Electron. Mater. 22, 1417 (1993).

${ }^{4}$ M. Kaminska and E. R. Weber, Mat. Sci. Forum. 83-87, 1033 (1992).

${ }^{5}$ F. W. Smith, Mater. Res. Soc. Symp. Proc. 241, 3 (1992).

${ }^{6}$ J. Dabrowski and M. Scheffler, Phys. Rev. Lett. 60, 2183 (1988).

${ }^{7}$ D. J. Chadi and K. J. Chang, Phys. Rev. Lett. 60, 2183 (1988).

${ }^{8}$ M. O. Manasreh, D. C. Look, K. R. Evans, and C. E. Stutz, Phys. Rev. B 41, 10272 (1990).

${ }^{9}$ X. Liu, A. Prasad, W. M. Chen, A. Kurpiewski, A. Stoschek, Z. LilientalWeber, and E. R. Weber, Appl. Phys. Lett. 65, 3002 (1994).

${ }^{10}$ J. Haruyama, N. Goto, and H. Negishi, Appl. Phys. Lett. 61, 928 (1992).

${ }^{11}$ M. Kaminska, M. Skowronski, J. Lagowski, J. M. Parsey, and H. C. Gatos, Appl. Phys. Lett. 43, 302 (1983).

${ }^{12}$ A. C. Warren, J. M. Woodall, J. L. Freeouf, D. Grichkowsky, D.T. McInturff, M. R. Melloch, and N. Otsuka, Appl. Phys. Lett. 57, 1331 (1990).

${ }^{13}$ E. Yablonovitch, T. Gmitter, J. P. Harbison, and R. Bhat, Appl. Phys. Lett. 51, 2222 (1987).

${ }^{14}$ G. C. DeSalvo, W. F. Tseng, and J. Comas, J. Electrochem. Soc. 139, 3 (1992).

${ }^{15}$ S. U. Dankowski, P. Kiesel, B. Knüpfer, M. Kneissl, G. H. Döhler, U. D. Keil, D. R. Dykaar, and R. F. Kopf, Appl. Phys. Lett. 65, 3271 (1994). 